\title{
Right axillary vein thrombosis due to malpositioning of a central venous catheter via the internal jugular vein
}

\author{
Ji Su Jang ${ }^{1}$, Han Joon Kim², Jae In Yoo ${ }^{1}$, Jae Jun Lee ${ }^{1}$, and So Young Lim ${ }^{1}$ \\ Departments of ${ }^{1}$ Anesthesiology and Pain Medicine, ${ }^{2}$ General Surgery, School of Medicine, Hallym University, Chuncheon, Korea
}

Central venous catheterization (CVC) is commonly used in the operative setting for central venous pressure monitoring, drug administration, and the rapid introduction of fluids. However, CVC can have many severe complications, even for experienced physicians. Furthermore, complications of CVC are increasingly being recognized as a major source of morbidity. We report a rare, but serious, case of right axillary vein thrombosis due to malpositioning of a central venous catheter tip following right internal jugular venous (IJV) catheterization.

A 67-year-old, 40-kg, 158-cm-tall female with cholangio- cellular carcinoma of the liver required CVC. With the patient supine, the neck was prepped and draped sterilely. The position of the IJV was determined using a 7.5- $\mathrm{MHz}$ linear ultrasound probe (M-Turbo; SonoSite, Bothell, WA, USA) and ultrasound (MicroMaxx; SonoSite). A central venous catheter (Two-Lumen Central Venous Catheterization Set with Blue FlexTip; Arrow, Reading, PA, USA) was advanced into the IJV. Subsequently, the aspiration of blood via the two lumens of the catheter was observed, and the catheter was secured at a depth of $15 \mathrm{~cm}$ below the skin. On connecting the transducer to the monitor,
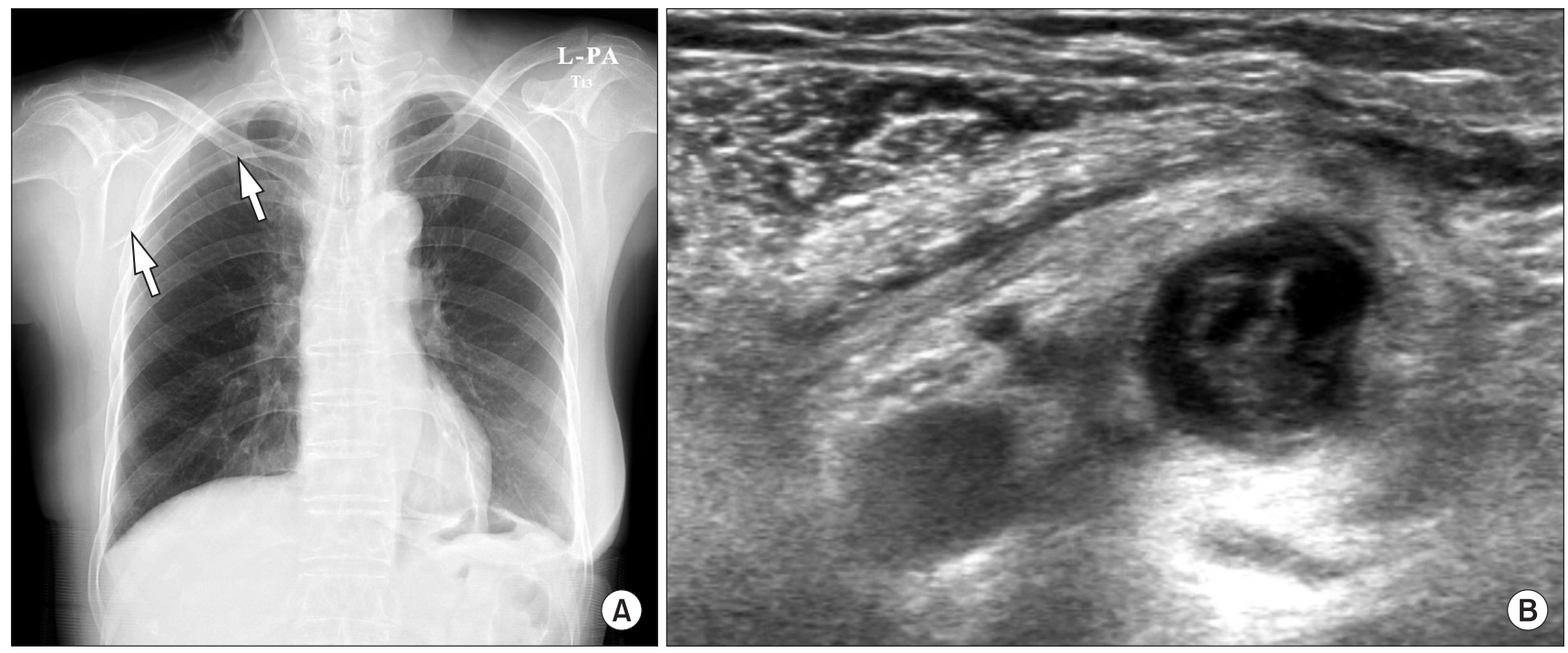

Fig. 1. (A) Plain chest radiograph showing the catheter tip location in the right axillary vein (white arrows). (B) Ultrasonography showing a thrombus in the right axillary vein.

Corresponding author: Jae Jun Lee, M.D., Department of Anesthesiology and Pain Medicine, Chuncheon Sacred Heart Hospital, 153, Kyo-dong, Chuncheon 200-704, Korea. Tel: 82-33-240-5594, Fax: 82-33-251-0941, E-mail: iloveu59@hallym.or.kr

(c) This is an open-access article distributed under the terms of the Creative Commons Attribution Non-Commercial License (http:// creativecommons.org/licenses/by-nc/3.0/), which permits unrestricted non-commercial use, distribution, and reproduction in any medium, provided the original work is properly cited. 
the opening central venous pressure (CVP) was noted as 7 $\mathrm{mmHg}$, and a waveform was observed. However, we did not carefully check whether the wave had an ideal CVP waveform. Six days after CVC, the patient complained of pain in the right upper arm, with swelling. On the chest radiograph, the terminus of the central venous catheter was located in the right axillary vein (Fig. 1A). An ultrasonographic examination showed vein thrombosis of the right axillary vein (Fig. 1B).

The traditionally preferred position of the central venous catheter tip is within the caudal third of the superior vena cava. However, central venous catheters placed without imaging guidance have a $10-30 \%$ incidence of malpositioning of the catheter tip in the right atrium [1]. When the central venous catheter is abnormally located in the right atrium, the risk for thrombosis increases [2]. Interestingly, the present rare case showed that the catheter tip could be abnormally located in the right axillary vein, and that this malpositioning could lead to thrombus formation. This complication should not be underestimated because it may be a source of pulmonary embolism. Malpositioning of a guidewire placed in the vein during CVC can be detected with a real-time ultrasonic examination. After the procedure, chest radiography should be performed to assess the location of the central venous catheter tip. These examinations can reduce the risk for thrombosis due to malpositioning. In addition, correct placement of the catheter tip is important for obtaining accurate CVP measurements. Inaccurate CVP measurements or the inability to obtain an ideal waveform is suggestive of an undesirable location of the catheter tip [3]. Therefore, as we did not carefully check the waveform to confirm the location of the tip, the CVP measurements of 7 to $9 \mathrm{mmHg}$ in this case might not have been correct.

A thrombus in the subclavian, axillar, or brachial vein is defined as an upper-extremity deep-vein thrombosis (UEDVT). Gbaguidi et al. [4] reported that the most common cause of UEDVT was central venous catheters $(62.2 \%)$, with malignancy being second $(27.2 \%)$. Thus, physicians should carefully consider these complications of CVC, especially in patients with malignancies and a high risk for thrombosis.

In conclusion, malpositioning of a central venous catheter can increase the risk for thrombosis, which may lead to pulmonary embolism. To detect the malpositioning of a central venous catheter tip, the location of the guidewire should be checked using ultrasonography during the procedure and chest radiography after the procedure, and the CVP waveform should be closely examined.

\section{References}

1. Vesely TM. Central venous catheter tip position: a continuing controversy. J Vasc Interv Radiol 2003; 14: 527-34.

2. Timsit JF, Farkas JC, Boyer JM, Martin JB, Misset B, Renaud B, et al. Central vein catheter-related thrombosis in intensive care patients: incidence, risk factors, and relationship with catheter-related sepsis. Chest 1998; 114: 207-13.

3. Agrawal P, Gupta B, D'souza N. Coiled central venous catheter in superior vena cava. Indian J Anaesth 2010; 54: 351-2.

4. Gbaguidi X, Janvresse A, Benichou J, Cailleux N, Levesque H, Marie I. Internal jugular vein thrombosis: outcome and risk factors. QJM 2011; 104: 209-19. 